\title{
杂交粳稻亲本产量性状优异配合力的标记基因型篮选
}

\author{
梁 奎 1 黄殿成 $1,2, * *$ 赵凯铭 ${ }^{1}$ 阮方松 1,3 谢 辉 1,4 马文霞 1 \\ 洪德林 1 ,
}

${ }^{1}$ 南京农业大学作物遗传与种质创新国家重点实验室, 江苏南京 $210095 ;{ }^{2}$ 中国农业科学院棉花研究所, 河南安阳 $455000 ;{ }^{3}$ 越南北中 部农业技术研究院, 越南义安荣市; ${ }^{4}$ 天津市水稻技术工程中心, 天津 300457

摘 要：提高杂交粳稻竞争优势的关键是改良其恢复系产量性状的配合力。为使之更富成效, 选用 115 个 SSR 引物 扩增 6 个粳稻 BT 型不育系和 12 个恢复系的标记基因型, 并按 NCII 遗传设计配制 72 个 $\mathrm{F}_{1}$ 组合, 分析 18 个亲本的 单株日产量、单株有效穗数、每穗总粒数、每穗实粒数、千粒重 5 个性状的配合力, 结合亲本 SSR 分子标记数据和 性状配合力数据筛选了 5 个性状优异配合力的标记基因型。结果发现 20 个 SSR 标记基因型与亲本产量及其构成性 状配合力显著相关。其中 8 个与亲本单个性状配合力相关; 6 个同时与亲本 2 个性状配合力相关; 4 个同时与亲本 3 个性状配合力相关; 2 个同时与亲本 4 个性状配合力相关。同时与多个性状配合力相关的标记基因型, 对各性状的作 用方向有正有负。RM152-165/170 是单株日产量和单株有效穗数优异配合力效应最大的标记基因型, 可使 $F_{1}$ 的相应 性状值增加 $20.6 \%$ 和 $12.7 \%$ 。优异配合力的标记基因型可直接用于粳稻恢复系配合力的分子标记辅助改良。

关键词：粳稻; 配合力; SSR 标记; 产量及其构成因素; 标记基因型

\section{Screening Marker Genotypes with Elite Combining Ability for Yield Traits in Parents of Hybrid Japonica Rice (Oryza sativa L.)}

LIANG Kui ${ }^{1}$, HUANG Dian-Cheng ${ }^{1,2, * *}$, ZHAO Kai-Ming ${ }^{1}$, NGUYEN Phuong-Tung ${ }^{1,3}$, XIE Hui ${ }^{1,4}$, MA Wen-Xia, and HONG De-Lin ${ }^{1, *}$

${ }^{1}$ State Key Laboratory of Crop Genetics and Germplasm Enhancement, Nanjing Agricultural University, Nanjing 210095, China; ${ }^{2}$ Cotton Research Institute, Chinese Academy of Agricultural Sciences, Anyang 455000, China; ${ }^{3}$ Agricultural Science Institute of Northern Central Vietnam, Nghe An-Vinh, Vietnam; ${ }^{4}$ Tianjin Subcentre of China National Hybrid Rice Research and Development Center, Tianjin 300457, China

\begin{abstract}
The area of japonica rice planted annually is 8280000 ha and accounts for $27 \%$ of total rice area in China. The area planting japonica hybrid rice is only $3 \%$ of the total area of japonica rice. The major reason for this situation is that competitive heterosis of hybrid cultivar is not conspicuous in yield, compared with conventional cultivar in japonica rice. The key factor of enhancing competitive heterosis of hybrid cultivar in japonica rice is to improve combining ability of yield related traits of restorer lines. In order to improve combining ability of restorer lines more efficiently, SSR marker genotypes with elite combining ability for five traits were screened in this study, by analyzing the data of combining ability and SSR markers in six CMS lines and twelve restorer lines that were genotyped using 115 pairs of SSR primers. Combining ability of the 18 parental lines was analyzed for daily yield per plant (DYP), panicles per plant (PP), total spikelets per panicle (TSP), filled spikelets per panicle (FSP) and 1000-grain weight (1000-GW), using the data of $72 \mathrm{~F}_{1} \mathrm{~s}$ made with $\mathrm{NC}$ II genetic design. Results showed that twenty SSR marker genotypes were significantly associated with combining ability of the five traits. Among them, eight for only one trait, six for two traits, four for three traits and two for four traits. Marker genotypes associated with combining ability of multiple traits increased or decreased trait value of $\mathrm{F}_{1}$. RM152-165/170 was a marker genotype of elite combining ability for PP and DYP with the maximum increasing effect, which increased $20.6 \%$ of DYP and $12.7 \%$ of PP in $F_{1}$ respectively. Those marker genotypes with increasing effect could be directly used to improve combining ability of restorer lines through the marker-assistant selection.
\end{abstract}

Keywords: Japonica rice; Combining ability; SSR; Yield and yield components; Marker genotypes

\footnotetext{
本研究由高等学校学科创新引智计划项目(B08025), 引进国际先进农业科学技术计划(948计划)项目(2006-G8[4]-31-1), 教育部科技基础条件平 台重点资助项目(505005)和国家高技术研究发展计划(863 计划)项目(2010AA101301)资助。

*通讯作者(Corresponding author): 洪德林, E-mail: delinhong@njau.edu.cn, Tel: 025-84396626

第一作者联系方式: E-mail: 2007101109@njau.edu.cn(梁奎); 2008201059@njau.edu.cn(黄殿成)＂ ${ }^{* *}$ 共同第一作者
}

Received(收稿日期): 2010-02-01; Accepted(接受日期): 2010-04-19. 
我国杂交粳稻经过 30 多年的研究, 已取得一定 的成绩, 但是相对于杂交籼稻的发展, 却显严重滞 后。杂交籼稻的种植面积已占中国水稻种植总面积 的 $57 \%$, 而杂交粳稻仅占 $3 \%^{[1]}$ 。杂交粳稻还有很大 的发展空间。杂交粳稻发展缓慢的原因是多方面的, 一是纯系粳稻品种的不断改良, 产量接近、米质优 于杂交粳稻组合, 让杂交粳稻的推广面临严峻的挑 战; 二是杂交粳稻育种中缺乏高配合力的恢复系, 导致杂交粳稻组合的竞争优势不强, 这是其根本原 因。这与早期杂交粳稻的育种基础有关。我国 1972 年引进 Boro II 细胞质后转育了一批粳稻不育系, 在 粳稻品种中未能找到有效恢复系, 而是通过籼粳杂 交, 后代再与粳稻复交的方法导入恢复基因, 选育 出含籼稻血缘的粳稻恢复系 ${ }^{[2-3]}$ 。目前使用的粳稻恢 复系都是从籼粳交后代中选育出来的, 由于在选育 过程中主要关注其恢复力的选择, 忽视了配合力的 选择, 导致粳稻恢复系配合力较低。选育高一般配 合力 (GCA)的粳稻恢复系是组配出优势杂交粳稻组 合的必要条件 ${ }^{[4]}$, 也是提高杂交粳稻竞争优势的必 由之路。

以往大量关于恢复系配合力的分析虽有助于鉴别 恢复系配合力的高低和提出杂种优势的配对模式 ${ }^{[5-11]}$, 但难于有效直接指导恢复系配合力的改良。分子标 记辅助选择已被广泛用于改良水稻目标性状 ${ }^{[12-14]}$, 而利用分子标记辅助选择的前提是必须篮选到与性 状基因紧密连锁的分子标记。配合力性状不同于普 通的形态性状, 必须通过杂合状态的 $F_{1}$ 群体才能测 定。因此, 篲选与配合力性状基因连锁的分子标记 有其特殊性, 必须与双列杂交相结合才能实现。刘 小川等 ${ }^{[15-18]}$ 曾对籼稻产量性状和米质性状配合力的 标记基因型进行过篮选, 并用篮选出的优异标记基 因型改良了籼稻恢复系明恢 63 的产量性状配合力。 在粳稻上尚无利用分子标记辅助改良恢复系产量性 状配合力的研究报道。本文报道利用 NCII 遗传设计 配制的 72 个组合 $\mathrm{F}_{1}$ 产量性状表型数据和两组亲本 SSR 分子标记基因型数据, 篮选出的杂交粳稻亲本 产量性状优异配合力的标记基因型结果, 为下一步 定向聚合有利等位变异、改良粳稻恢复系配合力打 下基础。

\section{1 材料与方法}

\section{1 试验材料}

选用武 3A、863A、6427A、9522A、六千辛 A、
武羌 $\mathrm{A}$ 等 6 个 BT 型不育系和 3726R-21、武育粳 R-39、6427R-16、157TR-68、4016LHR-69、秀水 04R、 C418、77302-1、C 堡、R254、湘晴、宁恢 8 号等 12 个恢复系, 以及用它们按 NCII 遗传设计配制的 72 个 $\mathrm{F}_{1}$ 杂种。这些不育系和恢复系都是生产上和育种 研究上常用的骨干亲本。

\section{2 田间种植和性状调查}

2007 年正季在南京农业大学江浦试验站水稻田 种植 6 个不育系、6 个保持系和 12 个恢复系。 5 月 7 日播种, 6 月 10 日移栽至大田。当季配制 72 个杂 交组合 $F_{1}$ 。其中有 16 个组合 $F_{1}$ 种子不足 100 粒, 同 年冬季在海南岛陵水南繁基地再做杂交补足。海南 冬季播栽期分别为 12 月 3 日和 1 月 5 日。2008 年 正季在南京农业大学江浦试验站种植 $\mathrm{F}_{1}$ 、亲本, 5 月 8 日将剥壳的 $\mathrm{F}_{1}$ 种子及亲本种子用 $50 \%$ 多菌灵粉剂 1000 倍液浸种, $24 \mathrm{~h}$ 后用清水冲洗干净, 5 月 10 日 将种子按编号播种到对应的培养血中发芽。5 月 18 日移到秩田寄栽。6 月 13 日移至大田单本栽插, 株 行距为 $17 \mathrm{~cm} \times 20 \mathrm{~cm}$, 每个小区种植 4 行, 每行 8 株。 完全随机区组排列, 3 次重复。栽培管理同大田。

成熟后，每个小区去边行调查 10 株计算单株有 效穗数, 实粒数在 5 粒以上的稻穗均记为有效穗。 然后每个小区去边行随机取 5 株的主茎穗(最高穗), 于室内考查穗总粒数、穗实粒数。风干后称千粒重, 3 次重复。将每个小区去边行收获脱粒风干后, 测小 区产量。以小区产量除以小区实收株数得单株平均 产量, 再除以全生育期得单株平均日产量。全生育 期是从播种至成熟期的天数。

\subsection{DNA 提取和 PCR 扩增及扩增产物电泳}

1.3.1 DNA 提取 2007 年在分䔉期剪取 12 份恢 复系和 6 份不育系的叶片, 按照文献[19-20]所述的 SDS 法提取总 DNA。

1.3.2 PCR 扩增 选用 115 对 SSR 引物对 18 个 亲本进行扩增。其中 62 对是文献报道的与控制产量 及其构成性状基因位点连锁的引物(表 1 , 此组简称 为 $\mathrm{A}$ 组); 其余 53 对是从实验室的现有引物中随机 选取的(此组简称为 $\mathrm{B}$ 组)。引物序列从水稻基因组 数据库中获得(http://www.gramene.org/)。引物由南 京金思特科技有限公司合成。 PCR 扩增体系 $10 \mu \mathrm{L}$, 含 $20 \mathrm{ng}^{2} \mathrm{~L}^{-1}$ 模板 DNA $1 \mu \mathrm{L} 、 25 \mathrm{mmol} \mathrm{L}^{-1} \mathrm{MgCl}_{2} 0.6$ $\mu \mathrm{L} 、 2 \mathrm{pmol} \mu \mathrm{L}^{-1}$ 前引物 $0.7 \mu \mathrm{L} 、 2 \mathrm{pmol} \mu \mathrm{L}^{-1}$ 后引物 $0.7 \mu \mathrm{L} 、 2.5 \mathrm{mmol} \mathrm{L}^{-1} \mathrm{dNTP} 0.2 \mu \mathrm{L} 、 10 \times \mathrm{SSR}$ 缓冲液

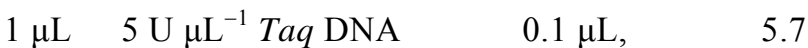


表 1 与水稻产量及其构成性状 QTL 连锁的 62 对 SSR 引物

Table 162 pairs of SSR markers linked with QTLs for yield and yield components in rice

\begin{tabular}{|c|c|c|c|}
\hline $\begin{array}{l}\text { 编号 } \\
\text { Code }\end{array}$ & $\begin{array}{c}\text { 染色体 } \\
\text { Chr. }\end{array}$ & $\begin{array}{l}\text { 连锁标记 } \\
\text { Marker linked }\end{array}$ & $\begin{array}{l}\text { 性状 } \\
\text { Trait }\end{array}$ \\
\hline 1 & 1 & RM1 & 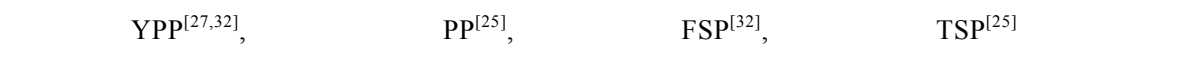 \\
\hline 2 & 1 & RM104 & 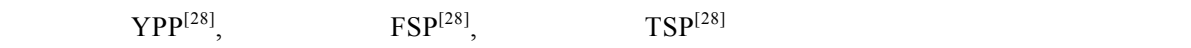 \\
\hline 3 & 1 & RM212 & 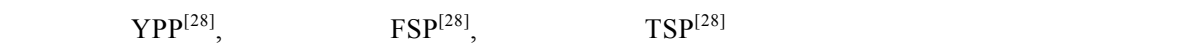 \\
\hline 4 & 1 & $\mathbf{R M}^{\mathbf{a}}$ & 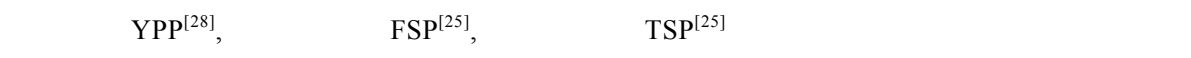 \\
\hline 5 & 1 & RM246 & 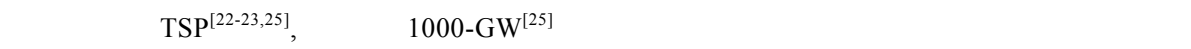 \\
\hline 6 & 1 & RM259 & $\begin{array}{l}\text { 单株产量 } \mathrm{YPP}^{[27-28]} \text {, 单株有效穗数 } \mathrm{PP}^{[21,25,27]} \text {, 每穗实粒数 } \mathrm{FSP}^{[24-25]} \text {, 每穗总粒数 } \mathrm{TSP}^{[22,25-26]} \text {, 千 } \\
\text { 粒重 } 1000-\mathrm{GW}^{[21]}\end{array}$ \\
\hline 7 & 1 & RM428 & 每穗总粒数 $\mathrm{TSP}^{[22]}$ \\
\hline 8 & 1 & $\mathbf{R M}^{\mathbf{a}}$ & 单株产量 $\mathrm{YPP}^{[21]}$, 千粒重 $1000-\mathrm{GW}^{[21]}$ \\
\hline 9 & 2 & RM207 & 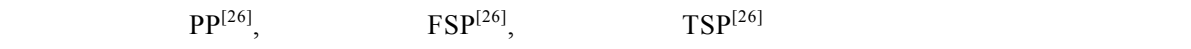 \\
\hline 10 & 2 & $\mathbf{R M}^{208} \mathbf{a}^{\mathrm{a}}$ & $\begin{array}{l}\text { 单株产量 } \mathrm{YPP}^{[30]} \text {, 单株有效穗数 } \mathrm{PP}^{[26]} \text {, 每穗实粒数 } \mathrm{FSP}^{[26,29]} \text {, 每穗总粒数 } \mathrm{TSP}^{[21,26,29]} \text {, 千粒重 } \\
1000-\mathrm{GW}^{[21]}\end{array}$ \\
\hline 11 & 2 & RM221 & 单株产量 $\mathrm{YPP}^{[28]}$ 、单株有效穗数 PPP ${ }^{[28]}$ 、每穗总粒数 TSP ${ }^{[28]}$ \\
\hline 12 & 2 & RM263 & $\begin{array}{l}\text { 单株产量 } \mathrm{YPP}^{[27-28]} \text {, 单株有效穗数 } \mathrm{PP}^{[28]} \text {, 每穗实粒数 } \mathrm{FSP}^{[28,32]} \text {, 每穗总粒数 } \mathrm{TSP}^{[28]} \text {, 千粒重 } \\
1000-\mathrm{GW}^{[22]}\end{array}$ \\
\hline 13 & 2 & RM290 & 千粒重 $1000-\mathrm{GW}^{[33]}$ \\
\hline 14 & 2 & $\mathbf{R M}^{341} 1^{\mathrm{a}}$ & 千粒重 $1000-\mathrm{GW}^{[33]}$ \\
\hline 15 & 2 & $\mathbf{R M} 475^{\mathrm{a}}$ & 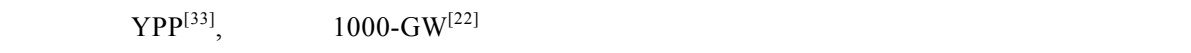 \\
\hline 16 & 3 & $\mathbf{R M 1 4 8}^{\mathrm{a}}$ & 单株产量 $\mathrm{YPP}^{[31]}$, 每穗总粒数 $\mathrm{TSP}^{[31]}$, 千粒重 $1000-\mathrm{GW}^{[28]}$ \\
\hline 17 & 3 & RM16 & 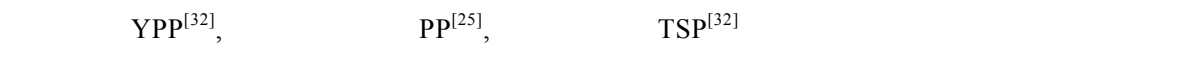 \\
\hline 18 & 3 & RM227 & 单株产量 $\mathrm{YPP}^{[31]}$, 每穗总粒数 $\mathrm{TSP}^{[23]}$ \\
\hline 19 & 3 & RM569 & 每穗总粒数 $\mathrm{TSP}^{[21]}$ \\
\hline 20 & 3 & RM570 ${ }^{\mathbf{a}}$ & 单株有效穗数 $\mathrm{PP} \mathrm{P}^{[25]}$ \\
\hline 21 & 3 & RM85 & 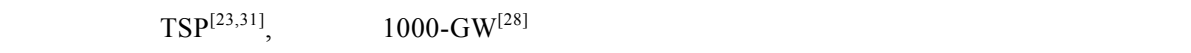 \\
\hline 22 & 4 & RM142 & 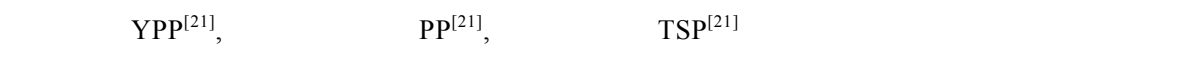 \\
\hline 23 & 4 & RM252 & 单株产量 $\mathrm{YPP}^{[32]}$, 每穗总粒数 $\mathrm{TSP}^{[32]}$ \\
\hline 24 & 4 & RM280 & 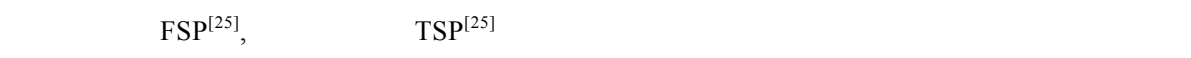 \\
\hline 25 & 4 & RM303 & 每穗实粒数 $\mathrm{FSP}^{[25]}$, 每穗总粒数 $\mathrm{TSP}^{[22,23,25]}$ \\
\hline 26 & 4 & RM317 & 每穗总粒数 $\mathrm{TSP}^{[22]}$ \\
\hline 27 & 5 & RM163 & 单株产量 $\mathrm{YPP}^{[31]}$, 单株有效穗数 $\mathrm{PP}^{[32]}$ \\
\hline 28 & 5 & RM164 & 单株有效穗数 $\mathrm{PP}^{[22]}$, 每穗实粒数 $\mathrm{FSP}^{[22]}$ \\
\hline 29 & 6 & RM19715 & 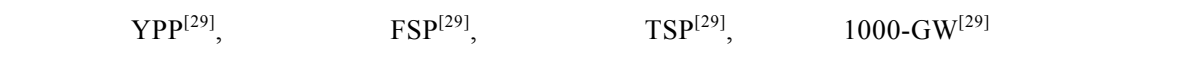 \\
\hline 30 & 6 & RM19784 & 单株产量 YPP ${ }^{[29]}$, 每穗实粒数 FSP ${ }^{[29]}$ \\
\hline 31 & 6 & RM204 & 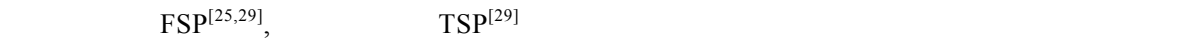 \\
\hline 32 & 6 & RM225 & 每穗总粒数 TSP ${ }^{[29]}$ \\
\hline 33 & 6 & RM253 & 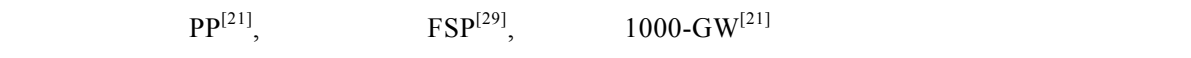 \\
\hline 34 & 6 & RM276 & 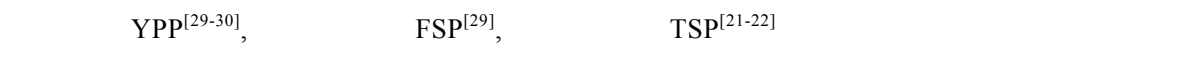 \\
\hline 35 & 6 & RM314 & 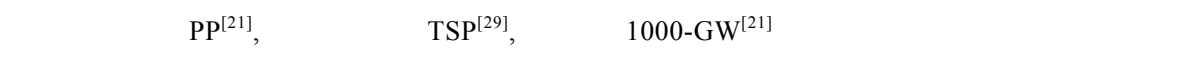 \\
\hline 36 & 6 & RM340 & 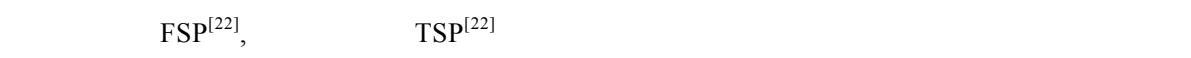 \\
\hline 37 & 6 & $\mathbf{R M} 345^{\mathrm{a}}$ & 单株有效穗数 $\mathrm{PP}^{[21]}$, 每穗实粒数 $\mathrm{FSP}^{[22]}$, 每穗总粒数 $\mathrm{TSP}^{[22]}$ \\
\hline 38 & 6 & RM402 & 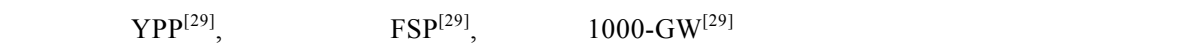 \\
\hline 39 & 6 & RM510 & 单株产量 $\mathrm{YPP}^{[29]}$, 每穗实粒数 $\mathrm{FSP}^{[29] ， \text { 每穗总粒数 TSP }}{ }^{[29]}$ \\
\hline 40 & 6 & RM549 & 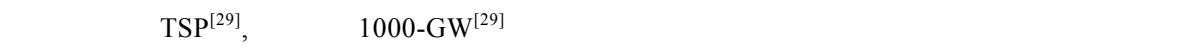 \\
\hline 41 & 6 & RM587 & 单株产量 $\mathrm{YPP}^{[29]}$ \\
\hline
\end{tabular}




\begin{tabular}{|c|c|c|c|}
\hline $\begin{array}{l}\text { 编号 } \\
\text { Code }\end{array}$ & $\begin{array}{l}\text { 染色体 } \\
\text { Chr. }\end{array}$ & $\begin{array}{c}\text { 连锁标记 } \\
\text { Marker linked }\end{array}$ & $\begin{array}{l}\text { 性状 } \\
\text { Trait }\end{array}$ \\
\hline 42 & 7 & RM10 & 每穗总粒数 $\mathrm{TSP}^{[32]}$ \\
\hline 43 & 7 & RM11 $^{\text {a }}$ & 单株产量 $\mathrm{YPP}^{[21,30]}$, 单株有效穗数 $\mathrm{PP}^{[21,30]}$, 每穗总粒数 $\mathrm{TSP}^{[32]}$, 千粒重 $1000-\mathrm{GW}^{[21]}$ \\
\hline 44 & 7 & RM234 & 单株产量 $\mathrm{YPP}^{[31]}$, 每穗总粒数 TSP $\mathrm{TP}^{[24]}$ 千粒重 $1000-\mathrm{GW}^{[25]}$ \\
\hline 45 & 7 & RM478 & 单株产量 $\mathrm{YPP}^{[31]}$ \\
\hline 46 & 7 & RM542 & 单株产量 $\mathrm{YPP}^{[27]}$, 每穗实粒数 $\mathrm{FSP}^{[27]}$ \\
\hline 47 & 8 & RM137 & 单株产量 $\mathrm{YPP}^{[31]}$ \\
\hline 48 & 8 & RM210 & 单株有效穗数 $\mathrm{PP}^{[32]}$, 每穗总粒数 $\mathrm{TSP}^{[31]}$ \\
\hline 49 & 8 & RM223 & 单株有效穗数 $\mathrm{PP}^{[32]}$, 每穗总粒数 $\mathrm{TSP}^{[25]}$ \\
\hline 50 & 8 & RM331 & 单株产量 $\mathrm{YPP}^{[25]}$, 每穗实粒数 $\mathrm{FSP}^{[25]}$, 每穗总粒数 $\mathrm{TSP}^{[25]}$ \\
\hline 51 & 8 & RM5556 & 单株产量 $\mathrm{YPP}^{[25]}$, 每穗实粒数 $\mathrm{FSP}^{[25]}$, 每穗总粒数 $\mathrm{TSP}^{[25]}$ \\
\hline 52 & 8 & RM72 & 单株产量 $\mathrm{YPP}^{[31]}$, 每穗总粒数 $\mathrm{TSP}^{[21]}$ \\
\hline 53 & 10 & RM228 & 单株产量 $\mathrm{YPP}^{[31]}$, 千粒重 $1000-\mathrm{GW}^{[28]}$ \\
\hline 54 & 10 & $\mathbf{R M}^{258^{\mathrm{a}}}$ & 单株产量 $\mathrm{YPP}^{[31]}$ \\
\hline 55 & 11 & RM167 & 单株有效穗数 $\mathrm{PP}^{[32]}$, 每穗总粒数 $\mathrm{TSP}^{[22]}$ \\
\hline 56 & 11 & RM224 & 单株有效穗数 $\mathrm{PP}^{[23]}$, 千粒重 $1000-\mathrm{GW}^{[21,33]}$ \\
\hline 57 & 11 & RM4B & 单株产量 $\mathrm{YPP}^{[32]}$, 每穗实粒数 $\mathrm{FSP}^{[32]}$, 每穗总粒数 $\mathrm{TSP}^{[32]}$ \\
\hline 58 & 12 & RM235 & 单株有效穗数 $\mathrm{PP}^{[30]}$, 每穗总粒数 $\mathrm{TSP}^{[21]}$, 每穗实粒数 $\mathrm{FSP}^{[31]}$ \\
\hline 59 & 12 & RM247 $^{\mathrm{a}}$ & 千粒重 $1000-G^{[22]}$ \\
\hline 60 & 12 & RM270 & 单株有效穗数 $\mathrm{PP}^{[30]}$, 每穗总粒数 $\mathrm{TSP}^{[21]}$ \\
\hline 61 & 12 & RM277 & 千粒重 $1000-\mathrm{GW}^{[28]}$ \\
\hline 62 & 12 & RM309 & 单株产量 $\mathrm{YPP}^{[31]}$ \\
\hline
\end{tabular}

粗体表示在 18 个亲本间扩增条带有差异的 SSR 引物; 粗体 ${ }^{a}$ 表示与配合力相关的 SSR 标记。

Bold letters represent polymorphic SSR markers among 18 parents; Bold letters with a represent SSR markers related to combining ability. YPP: yield per plant; TSP: total spikelets per panicle; FSP: filled spikelets per panicle; 1000-GW: 1000-grain weight; PP: panicles per plant.

$\mu \mathrm{L}$ 。扩增反应在 PTC-100 Peltier Thermal Cycler (MJ Research Inc., USA)上进行。PCR 程序为 $95^{\circ} \mathrm{C}$ 预变性 $5 \mathrm{~min} ; 95^{\circ} \mathrm{C} 30 \mathrm{~s}, 55^{\circ} \mathrm{C} 30 \mathrm{~s}, 72^{\circ} \mathrm{C} 1 \mathrm{~min}, 35$ 个循环; 最后 $72^{\circ} \mathrm{C}$ 延伸 $7 \mathrm{~min}$ 。

1.3.3 电泳、银染和记录扩增产物经 $8.0 \%$ 聚丙 烯酰胺凝胶 $(\mathrm{PAG})$ 电泳后, 以含 $10 \%$ 乙醇和 $0.5 \%$ 冰 乙酸的固定液固定凝胶 $12 \mathrm{~min}$; 以 $0.2 \% \mathrm{AgNO}_{3}$ 溶 液渗透 $12 \mathrm{~min}$; 以 $\mathrm{ddH}_{2} \mathrm{O}$ 清洗 2 次, 每次 $15 \mathrm{~s}$; 最后 用含 $15 \mathrm{~g} \mathrm{~L}^{-1} \mathrm{NaOH}$ 的 $1 \%$ 甲醛溶液显色, 待条带清 晰时取出放在灯箱上, 根据带型及 DNA 分子量标 记，记录每个扩增片段的碱基数。

\section{4 数据分析}

1.4 .1 亲本性状配合力分析 以小区的平均值为 单位, 按莫惠栋的 $p \times q$ 交配模式进行亲本性状的配 合力分析 ${ }^{[34-35]}$, 同时参考徐辰武 ${ }^{[36]}$ 的总秩次方法对 亲本进行综合评价, 即先计算出各亲本产量及其构 成性状的一般配合力 (GCA)效应和特殊配合力方差 $\left(V_{\mathrm{SCA}}\right)$ ，然后根据各值大小给以不同秩次。对于每穗
总粒数、每穗实粒数、千粒重、单株有效穗数、单 株产量 5 个性状, GCA 秩次按从大到小排序, 值愈 大秩次愈小，越好的亲本其秩次愈小。 $V_{\mathrm{SCA}}$ 按从大 到小排序，值愈大秩次愈小。最后以总秩次作为亲 本的评价指标。

\subsection{2 亲本性状优异配合力的标记基因型篮选} 假定 SSR 引物 RM1 在 18 个亲本(6 个不育系和 12 个恢复系)之间扩增出 2 个条带, DNA 分子量分别为 $100 \mathrm{bp}$ 和 $200 \mathrm{bp}$ 。如果在该位点上, 某个不育系和 某个恢复系都携带 $100 \mathrm{bp}$ 或 $200 \mathrm{bp}$ 的等位条带，则 推定它们配组的 $F_{1}$ 代在这个位点上是 $100 \mathrm{bp}$ 或 200 $\mathrm{bp}$ 的纯合体。如果在该位点上, 某个不育系携带 100 $b p$ 的等位条带, 某个恢复系携带 $200 \mathrm{bp}$ 的等位条带, 则推定它们配组的 $F_{1}$ 代在这个位点上是 $100 \mathrm{bp}$ 和 $200 \mathrm{bp}$ 的杂合体。根据 $\mathrm{F}_{1}$ 代在这个位点上是杂合体 还是纯合体, 将 72 个组合分为两组, 凡在这个位点 上是杂合体的组合归为杂合组，在这个位点上是纯 合体的组合归为纯合组, 纯合组包括 $100 \mathrm{bp}$ 纯合体 
组合和 $200 \mathrm{bp}$ 纯合体组合。将“杂合组”的性状平均 值与“纯合组”的性状平均值进行差异显著性 $t$ 测验。 若差值为正, 且 $t$ 值显著或极显著, 则表明标记基因 型 RM1-100 bp/200 bp (简写为 RM1-100/200)为配合 力增效标记基因型; 若差值为负, 且 $t$ 值显著或极显 著, 则表明 RM1-100/200 为配合力减效标记基因型。 若育种目标要求性状值越大越好, 则前者为优异配 合力的标记基因型; 反之, 后者为优异配合力的标 记基因型。如果 SSR 引物 RM1 在这 18 个亲本之间 扩增出 3 个条带(100 bp、200 bp 和 300 bp), 则对扩 增条带进行两两计算，即分别对 RM1-100/200、 RM1-100/300、RM1-200/300 三种标记基因型进行 运算。当对 $100 \mathrm{bp}$ 和 $300 \mathrm{bp}$ 两条带进行计算时, 凡 是 $F_{1}$ 代在这个 RM1 位点上是杂合体(即 RM1-100/ 300)的组合均归为 “杂合组”，其余组合均归为“纯合 组”。如果 SSR 引物 RM1 在这 18 个亲本之间扩增 出 4 个及以上条带, 计算方法同 3 个条带。优异配 合力标记基因型篮选的计算工作, 由作者运用 MATLAB 语言编制的程序 CAScreen 1.0 运算完成。

\section{2 结果与分析}

2.16 个不育系和 12 个恢复系 5 个性状一般配合 力分析

对 72 个组合 3 次重复的随机区组试验结果进行 方差分析表明, 产量及其构成性状组合间差异均达 到显著水平(表略)。可以对这 5 个性状进行亲本一般 配合力效应的估算和显著性测验。

由表 2 可知, 不育系中，单株日产量 GCA 效应 最大的是 BT-六千辛A; 单株有效穗数 GCA 效应最 大的是 $\mathrm{BT}-$ 六千辛 $\mathrm{A}$; 每穗总粒数和每穗实粒数 GCA 效应最大的是 BT-863A; 千粒重 GCA 效应最大 的是 BT-9522A。恢复系中, 单株日产量 GCA 效应 最大是 157TR-68; 单株有效穗数 GCA 效应最大的 是 3726R-21; 每穗总粒数和每穗实粒数 GCA 效应 最大的是 77302-1; 千粒重 GCA 效应最大的是秀水 04R。综合 5 个性状，根据 GCA 效应总秩次排序，同 时参考 $V_{\mathrm{SCA}}$ 总秩次排序, BT-863A、BT-武 3A、BT六千辛 $\mathrm{A}$ 是较优不育系, 其中 BT- $863 \mathrm{~A}$ 最优; $157 \mathrm{TR}-$

表 218 个亲本 5 个性状的一般配合力效应及其显著性检验和综合评价

Table 2 GCA effect and their significance test of five traits and integrative evaluation in 18 parents

\begin{tabular}{|c|c|c|c|c|c|c|c|c|c|}
\hline $\begin{array}{l}\text { 亲本 } \\
\text { Parent }\end{array}$ & $\begin{array}{c}\text { 单株 } \\
\text { 日产量 } \\
\text { DYP (g) }\end{array}$ & $\begin{array}{c}\text { 单株有效 } \\
\text { 穗数 } \\
\text { PP }\end{array}$ & $\begin{array}{c}\text { 每穗 } \\
\text { 总粒数 } \\
\text { TSP }\end{array}$ & $\begin{array}{c}\text { 每穗 } \\
\text { 实粒数 } \\
\text { FSP }\end{array}$ & $\begin{array}{c}\text { 千粒重 } \\
\text { 1000-GW } \\
(\mathrm{g})\end{array}$ & $\begin{array}{c}\text { GCA 总秩次 } \\
\text { Total rank of } \\
\text { GCA }\end{array}$ & $\begin{array}{c}\text { 排序 } \\
\text { Sequence }\end{array}$ & $\begin{array}{c}V_{\mathrm{SCA}} \text { 总秩次 } \\
\text { Total rank of } \\
V_{\mathrm{SCA}}\end{array}$ & $\begin{array}{c}\text { 排序 } \\
\text { Sequence }\end{array}$ \\
\hline Wu 3A & 0.001 & $-0.35^{* *}$ & -0.99 & -2.88 & 0.03 & 15 & 1 & 23 & 4 \\
\hline $863 \mathrm{~A}$ & $-0.01^{*}$ & $-0.64^{* *}$ & $10.25^{* *}$ & $8.00^{* *}$ & 0.03 & 15 & 1 & 10 & 1 \\
\hline $6427 \mathrm{~A}$ & -0.001 & -0.22 & 4.06 & $6.21^{* *}$ & $-0.66^{* *}$ & 16 & 2 & 22 & 3 \\
\hline $9522 \mathrm{~A}$ & -0.001 & $-0.77^{* *}$ & -0.60 & -3.32 & $1.58^{* *}$ & 17 & 3 & 20 & 2 \\
\hline Liuqianxin A & $0.03^{* *}$ & $1.38^{* *}$ & -1.80 & -3.65 & $-0.85^{* *}$ & 18 & 4 & 10 & 1 \\
\hline Wuqiang A & $-0.02^{* *}$ & $0.60^{* *}$ & $-10.92^{* *}$ & -4.35 & -0.13 & 24 & 5 & 20 & 2 \\
\hline 3726R-21 & -0.01 & $1.20^{* *}$ & $-22.98^{* *}$ & $-19.94^{* *}$ & -0.76 & 39 & 8 & 49 & 9 \\
\hline Wuyujing R-39 & $-0.02^{*}$ & $1.11^{* *}$ & $-59.86^{* *}$ & $-41.58^{* *}$ & $0.51^{* *}$ & 44 & 11 & 42 & 8 \\
\hline $6427 \mathrm{R}-16$ & $-0.03^{* *}$ & $0.73^{* *}$ & $-19.30^{* *}$ & $-8.09^{*}$ & -0.22 & 31 & 4 & 28 & 3 \\
\hline 157TR-68 & $0.08^{* *}$ & 0.23 & $11.80^{* *}$ & $19.08^{* *}$ & -0.98 & 16 & 1 & 31 & 5 \\
\hline 4016LHR-69 & $-0.03^{* *}$ & $-0.45^{* *}$ & 2.22 & -5.05 & -1.11 & 43 & 10 & 29 & 4 \\
\hline Xiushui 04R & $0.03^{* *}$ & $0.43^{* *}$ & $-27.20^{* *}$ & $-20.81^{* *}$ & $2.21^{* *}$ & 36 & 7 & 41 & 7 \\
\hline C418 & 0.001 & $-0.96^{* *}$ & $19.02^{* *}$ & $7.04^{*}$ & $1.64^{* *}$ & 22 & 2 & 31 & 5 \\
\hline $77302-1$ & 0.001 & $-0.42^{*}$ & $53.18^{* *}$ & $33.23^{* *}$ & -1.73 & 28 & 3 & 21 & 2 \\
\hline $\mathrm{C}$ bao & $-0.02^{* *}$ & -0.27 & 1.43 & -3.34 & $1.17^{* *}$ & 41 & 9 & 28 & 3 \\
\hline R254 & $-0.02^{* *}$ & $-0.69^{* *}$ & $12.07^{* *}$ & 4.63 & $-0.40^{*}$ & 33 & 5 & 17 & 1 \\
\hline Xiangqin & -0.01 & $-0.48^{* *}$ & $18.48^{* *}$ & $17.15^{* *}$ & -0.99 & 35 & 6 & 32 & 6 \\
\hline Ninghui 8 & $0.03^{* *}$ & $-0.44^{* *}$ & $11.13^{* *}$ & $17.68^{* *}$ & $0.67^{* *}$ & 22 & 2 & 41 & 7 \\
\hline
\end{tabular}

"表示 $5 \%$ 水平差异显著; ${ }^{* *}$ 表示 $1 \%$ 水平差异显著。

": significant at $5 \%$ probability level; ${ }^{* *}$ : significant at $1 \%$ probability level. DYP: daily yield per plant; PP: panicles per plant; TSP: total spikelets per panicle; FSP: filled spikelets per panicle; 1000-GW: 1000-grain weight. 
68、R254、C418、宁恢 8 号是较优恢复系, 其中 157TR-68 最优。

2.2 亲本 5 个性状优异配合力的标记基因型篮选 在 115 对 SSR 引物中, 只有 59 对引物在 18 个 亲本间扩增出有差异的条带, 其中 35 对来自 $\mathrm{A}$ 组引 物(表 1)。59 个多态位点在 18 个亲本间共检测到 153 个等位变异, 每个位点平均为 2.59 个, 变异幅度为 $2-$ 5 个。59 个多态性 SSR 位点按照 CAScreen 1.0 篮选 程序计算, 与亲本 5 个性状配合力显著相关的标记 基因型共有 42 个(表 3), 其中 25 个为亲本性状优异 配合力的标记基因型，17 个为亲本性状不良配合力 的标记基因型。

筛选到 7 个标记基因型与亲本单株日产量配合 力显著相关, 其中 4 个是增效的, 范围在 $11.1 \%$ 20.6\%之间。增效标记基因型 RM152-165/170 可以使 $F_{1}$ 单株日产量平均增加 $20.62 \%$ (表 3)。携带 RM152 $165 \mathrm{bp}$ 等位变异的载体亲本有六千辛 A、157TR-68; 携带 RM152-170 bp 等位变异的载体亲本有武 3A、 863A、6427A、9522A、武羌 A、3726R-21、武育粳 R-39、6427R-16、4016LHR-69、秀水 04R、77302-1、 C 堡、R254、湘晴、宁恢 8 号。3 个标记基因型是 减效的, 范围在 $-9.1 \% \sim-13.3 \%$ 之间, 减效标记基 因型 RM148 145/150 可使 $F_{1}$ 单株日产量平均减少 13.3\% (表 3)。携带 RM148-145 bp 等位变异的载体 亲本有 3726R-21、武育粳 R-39、6427R-16、C418、 77302-1、C 堡、R254、湘晴。携带 RM148-150 bp 等位变异的载体亲本有 157TR-68、4016LHR-69、秀 水 $04 \mathrm{R} 、$ 宁恢 8 号、武 $3 \mathrm{~A} 、 863 \mathrm{~A} 、 6427 \mathrm{~A} 、 9522 \mathrm{~A}$ 、 六千辛 $\mathrm{A}$ 、武美 $\mathrm{A}$ 。

篮选到 12 个标记基因型与亲本每穗总粒数配 合力显著相关, 其中 10 个是增效的, 范围在 $10.3 \%$ 26.9\%之间，效应最大标记基因型是 RM341-150/180 (表 3); 2 个是减效的。

篮选到 10 个标记基因型与亲本每穗实粒数配 合力显著相关，其中 7 个是增效的，范围在 $10.8 \%$ $20.3 \%$ 之间; 3 个是减效的, 范围在 $-9.0 \% \sim-13.7 \%$ 之 间(表 3)。

篮选到 4 个标记基因型与亲本千粒重配合力显 著相关, 其中 2 个是增效的(表 3)。RM5-110/115 和 RM258-142/165 可以分别增加 $F_{1}$ 千粒重的 $7.7 \%$ 和 5.1\%。RM340-120/171 和 RM3-120/150 两个标记基 因型是减效的，分别减少 $\mathrm{F}_{1}$ 千粒重的 $4.8 \%$ 和 $6.2 \%$ 。 篮选到 9 个标记基因型与亲本单株有效穗数配
合力显著相关，其中 2 个是增效的; 7 个是减效的，范 围在 $-8.9 \%$ - $15.5 \%$ 之间(表 3)。减效标记基因型 RM475-200/250 可以减少 $F_{1}$ 杂种 $15.5 \%$ 的单株有效 穗数。

\section{3 与亲本多个性状配合力相关的标记基因型}

表 3 所列的 42 个标记基因型中, 存在同一个标 记基因型与亲本多个性状配合力相关的现象。根据 一个标记基因型与亲本配合力相关的性状个数, 把 表 3 的结果进行归类列于表 4 。表 4 显示, 有 2 个标 记基因型同时与亲本 4 个性状配合力相关; 有 6 个 标记基因型同时与亲本 3 个性状配合力相关; 有 4 个标记基因型同时与亲本 2 个性状配合力相关; 有 8 个标记基因型与亲本单个性状配合力相关。此外, 每穗总粒数、每穗实粒数的亲本配合力标记基因型 的效应与单株有效穗数的亲本配合力标记基因型的 效应相反，如 RM340-120/171、RM7120-170/180、 RM11-131/147、RM341-150/180 等 7 个标记基因型; 千粒重的亲本配合力标记基因型的效应与每穗总粒 数、每穗实粒数的亲本配合力标记基因型的效应相 反，如标记基因型 RM340-120/171 和 RM3-120/150; 单株有效穗数亲本配合力标记基因型的效应与单株 日产量的亲本配合力标记基因型的效应一致, 如标 记基因型 RM7120-170/180 和 RM152-165/170。标记 基因型 RM7120-170/180 增加 $F_{1}$ 的每穗总粒数和每 穗实粒数, 但由于减少 $\mathrm{F}_{1}$ 单株有效穗数, 最终导致 $\mathrm{F}_{1}$ 单株日产量的降低, 这说明对于提高杂交粳稻的 产量, 提高单株有效穗数显得尤为重要。

归类后的 20 个标记基因型中有 15 个来源于 A 组引物(表 4), 占 $75 \%$; 来源于 B 组的仅有 5 个, 占 $25 \%$ 。这说明试验开始前有目的地选择 SSR 引物可 以提高亲本性状配合力标记基因型的篎选效率。

\section{3 讨论}

在利用本研究篮选到的这 20 个标记基因型时, 要注意同一标记基因型与亲本配合力相关的性状数 目。对于一个标记基因型只与一个性状配合力相关 的, 可直接根据标记基因型进行辅助选择改良。对 于与亲本多个性状配合力相关的标记基因型, 要考 虑性状之间配合力效应的方向。有些标记基因型与 多个性状配合力的方向是一致的, 有些则相反。例 如标记基因型 RM152-165/170 同时与单株有效穗数 和单株日产量配合力相关, 且都是正方向的, 利用 RM152-165/170 辅助选择, 可对这 2 个性状的亲本 
表 3 与亲本单个性状配合力相关的标记基因型

Table 3 Marker genotypes significantly related to combining ability of each trait in parents

\begin{tabular}{|c|c|c|c|c|c|c|c|}
\hline \multirow[b]{2}{*}{$\begin{array}{l}\text { 性状 } \\
\text { Trait }\end{array}$} & \multirow[b]{2}{*}{$\begin{array}{c}\text { 标记基因型 } \\
\text { Marker genotype }\end{array}$} & \multicolumn{2}{|c|}{ 杂合组 Heterozygous group } & \multicolumn{2}{|c|}{ 纯合组 Homozygous group } & \multirow{2}{*}{$\begin{array}{c}\text { 增减率 } \\
\text { Percentage of } \\
\text { increase or } \\
\text { decrease }(\%)\end{array}$} & \multirow[b]{2}{*}{$\begin{array}{c}t \text { 值 } \\
t \text {-value }\end{array}$} \\
\hline & & $\begin{array}{c}\mathrm{F}_{1} \text { 组合数 } \\
\text { Number of } \\
\mathrm{F}_{1} \text { cross }\end{array}$ & $\begin{array}{l}\text { 各 } \mathrm{F}_{1} \text { 组合平均数 } \\
\text { Average of } \mathrm{F}_{1} \text { 's }\end{array}$ & $\begin{array}{c}\mathrm{F}_{1} \text { 组合数 } \\
\text { Number of } \\
\mathrm{F}_{1} \text { cross }\end{array}$ & $\begin{array}{l}\text { 各 } \mathrm{F}_{1} \text { 组合平均数 } \\
\text { Average of } \mathrm{F}_{1} \text { 's }\end{array}$ & & \\
\hline \multirow{7}{*}{$\begin{array}{c}\text { 单株日产量 } \\
\text { DYP }\end{array}$} & RM152-165/170 & 15 & 0.33 & 57 & 0.27 & 20.6 & 5.02 \\
\hline & RM570-207/257 & 12 & 0.32 & 60 & 0.28 & 16.8 & 3.55 \\
\hline & RM19715-160/170 & 16 & 0.31 & 56 & 0.28 & 13.3 & 3.08 \\
\hline & RM23-150/160 & 30 & 0.30 & 42 & 0.27 & 11.2 & 3.00 \\
\hline & RM148-145/150 & 48 & 0.27 & 24 & 0.31 & -13.3 & -4.13 \\
\hline & RM5639-150/160 & 32 & 0.27 & 40 & 0.30 & -10.2 & -3.02 \\
\hline & RM7120-170/180 & 36 & 0.27 & 36 & 0.30 & -9.1 & -2.67 \\
\hline \multirow{12}{*}{$\begin{array}{c}\text { 每穗总粒数 } \\
\text { TSP }\end{array}$} & RM341-150/180 & 48 & 229.11 & 24 & 180.61 & 26.7 & 8.92 \\
\hline & RM1211-150/160 & 36 & 233.89 & 36 & 191.99 & 21.8 & 7.51 \\
\hline & RM11-131/147 & 42 & 229.73 & 30 & 189.43 & 21.3 & 6.85 \\
\hline & RM340-120/171 & 36 & 232.40 & 36 & 193.48 & 20.1 & 6.63 \\
\hline & RM7120-170/180 & 36 & 230.67 & 36 & 195.21 & 18.2 & 5.74 \\
\hline & RM475-200/250 & 35 & 230.77 & 37 & 196.07 & 17.7 & 5.56 \\
\hline & RM3-120/150 & 24 & 234.43 & 48 & 202.20 & 15.9 & 4.64 \\
\hline & RM23-150/160 & 30 & 226.72 & 42 & 203.10 & 11.6 & 3.35 \\
\hline & RM345-160/172 & 30 & 225.43 & 42 & 204.02 & 10.5 & 2.99 \\
\hline & RM208-185/175 & 21 & 228.02 & 51 & 206.73 & 10.3 & 2.72 \\
\hline & RM208-175/180 & 17 & 192.04 & 55 & 219.40 & -12.5 & -3.34 \\
\hline & RM475-220/250 & 12 & 183.17 & 60 & 218.89 & -16.3 & -3.92 \\
\hline \multirow{10}{*}{$\begin{array}{c}\text { 每穗实粒数 } \\
\text { FSP }\end{array}$} & RM1211-150/160 & 36 & 195.47 & 36 & 162.53 & 20.3 & 7.63 \\
\hline & RM341-150/180 & 48 & 190.30 & 24 & 156.39 & 21.7 & 7.23 \\
\hline & RM340-120/171 & 36 & 191.68 & 36 & 166.32 & 15.3 & 5.08 \\
\hline & RM475-200/250 & 35 & 191.66 & 37 & 167.02 & 14.8 & 4.88 \\
\hline & RM11-131/147 & 42 & 189.19 & 30 & 164.73 & 14.9 & 4.75 \\
\hline & RM7120-170/180 & 36 & 187.94 & 36 & 170.06 & 10.6 & 3.29 \\
\hline & RM3-120/150 & 24 & 191.49 & 48 & 172.75 & 10.9 & 3.24 \\
\hline & RM247-160/172 & 20 & 167.12 & 52 & 183.57 & -9.0 & -2.65 \\
\hline & RM208-175/180 & 17 & 165.48 & 55 & 183.18 & -9.7 & -2.71 \\
\hline & RM475-220/250 & 12 & 158.10 & 60 & 183.18 & -13.7 & -3.46 \\
\hline \multirow{4}{*}{$\begin{array}{c}\text { 千粒重 } \\
1000-\mathrm{GW}\end{array}$} & RM5-110/115 & 15 & 26.40 & 57 & 24.52 & 7.7 & 4.42 \\
\hline & RM258-142/165 & 18 & 25.85 & 54 & 24.60 & 5.1 & 2.93 \\
\hline & RM340-120/171 & 36 & 24.31 & 36 & 25.50 & -4.7 & -3.26 \\
\hline & RM3-120/150 & 24 & 23.85 & 48 & 25.44 & -6.2 & -4.29 \\
\hline \multirow{9}{*}{$\begin{array}{c}\text { 单株有效穗 } \\
\text { PP }\end{array}$} & RM152-165/170 & 15 & 10.34 & 57 & 9.17 & 12.7 & 3.60 \\
\hline & RM208-175/180 & 17 & 10.13 & 55 & 9.19 & 10.2 & 2.95 \\
\hline & RM208-185/175 & 21 & 8.81 & 51 & 9.66 & -8.9 & -2.88 \\
\hline & RM1211-150/160 & 36 & 8.96 & 36 & 9.87 & -9.3 & -3.47 \\
\hline & RM340-120/171 & 36 & 8.95 & 36 & 9.87 & -9.3 & -3.47 \\
\hline & RM7120-170/180 & 36 & 8.87 & 36 & 9.96 & -10.9 & -4.25 \\
\hline & RM341-150/180 & 48 & 8.98 & 24 & 10.28 & -12.7 & -4.98 \\
\hline & RM11-131/147 & 42 & 8.89 & 30 & 10.15 & -12.5 & -5.12 \\
\hline & RM475-200/250 & 35 & 8.60 & 37 & 10.18 & -15.5 & -7.33 \\
\hline
\end{tabular}

$t=2.64(P=0.01)$. DYP: daily yield per plant; TSP: total spikelets per panicle; FSP: filled spikelets per panicle; 1000-GW: 1000-grain weight; PP: panicles per plant. 
表 4 与亲本多个性状配合力相关的标记基因型及对 $\mathbf{F}_{1}$ 性状值的增减率

Table 4 Marker genotypes related to combining ability of traits in parents and percentage of increase or decrease to trait value in $F_{1}(\%)$

\begin{tabular}{|c|c|c|c|c|c|c|}
\hline $\begin{array}{c}\text { 相关性状个数 } \\
\text { No. of traits related }\end{array}$ & $\begin{array}{c}\text { 标记基因型 } \\
\text { Marker genotype }\end{array}$ & $\begin{array}{c}\text { 单株有效穗数 } \\
\text { PP }\end{array}$ & $\begin{array}{l}\text { 每穗总粒数 } \\
\text { TSP }\end{array}$ & $\begin{array}{l}\text { 每穗实粒数 } \\
\text { FSP }\end{array}$ & $\begin{array}{c}\text { 千粒重 } \\
1000-\mathrm{GW}(\mathrm{g})\end{array}$ & $\begin{array}{c}\text { 单株日产量 } \\
\text { DYP (g) }\end{array}$ \\
\hline \multirow[t]{2}{*}{4} & RM340-120/171 & -9.3 & 20.1 & 15.3 & -4.7 & \\
\hline & RM7120-170/180 & -10.9 & 18.2 & 10.5 & & -9.1 \\
\hline \multirow[t]{6}{*}{3} & RM11-131/147 & -12.5 & 21.3 & 14.9 & & \\
\hline & RM208-175/180 & 10.2 & -12.5 & -9.7 & & \\
\hline & RM3-120/150 & & 15.9 & 10.8 & -6.2 & \\
\hline & RM341-150/180 & -12.7 & 26.9 & 21.7 & & \\
\hline & RM1211-150/160 & -9.3 & 21.8 & 20.3 & & \\
\hline & RM475-200/250 & -15.5 & 17.7 & 14.8 & & \\
\hline \multirow[t]{4}{*}{2} & RM152-165/170 & 12.7 & & & & 20.6 \\
\hline & RM208-185/175 & -8.9 & 10.3 & & & \\
\hline & RM23-150/160 & & 11.6 & & & 11.2 \\
\hline & RM475-220/250 & & -16.3 & -13.7 & & \\
\hline \multirow[t]{8}{*}{1} & RM148-145/150 & & & & & -13.3 \\
\hline & RM19715-160/170a & & & & & 13.3 \\
\hline & $\mathrm{RM} 247-160 / 172^{\mathrm{a}}$ & & & -9.0 & & \\
\hline & RM258-142/165 & & & & 5.1 & \\
\hline & RM345-160/172 ${ }^{\mathrm{a}}$ & & 10.5 & & & \\
\hline & RM5-110/115 & & & & 7.7 & \\
\hline & RM5639-150/160 & & & & & -10.2 \\
\hline & RM570-207/257 & & & & & 16.8 \\
\hline
\end{tabular}

表示来源于 $\mathrm{A}$ 组引物的标记基因型, 其余的来源于 B 组引物的标记基因型。

${ }^{a}$ indicates marker genotypes originated from SSR markers of A group, others originated from SSR markers of B group. PP: panicles per plant; TSP: total spikelets per panicle; FSP: filled spikelets per panicle; 1000-GW: 1000-grain weight; DYP: daily yield per plant.

配合力同时进行改良。同样, 利用 RM23-150/160 可 对每穗总粒数和单株日产量的配合力同时进行改 良。而标记基因型 RM208-185/175 对单株有效穗的 配合力效应是负值, 对每穗总粒数的配合力效应是 正值，两者方向相反。在亲本性状配合力改良中应 避免选用与每穗总粒数和单株有效穗数配合力同时 相关又方向相反的标记基因型, 而应选用分别对这 2 个性状配合力有利的标记基因型进行改良, 再通过 聚合来获得这 2 个性状配合力协调的优异亲本。

本研究结果显示, $863 \mathrm{~A}$ 是综合评价最优的不 育系; 157TR-68、R254、C418、宁恢 8 号是比较优 秀的恢复系, 在育种上均有较好的利用价值。86 优 8 号是 $863 \mathrm{~A}$ 和宁恢 8 号配组的三系杂交粳稻审定推 广组合 ${ }^{[37]}$ 。若进一步提高宁恢 8 号产量性状的配合 力, 改良恢复系仍与 863A 配组, 则可从 157TR-68 导入 RM152-170 bp 条带改良其单株有效穗数、单株 日产量的亲本配合力; 导入 RM23-160 bp 条带改良
其每穗总粒数、单株日产量的亲本配合力; 导入 RM570-257 bp 条带改良其单株日产量的亲本配合 力; 导入 RM345-172 bp 条带改良其每穗总粒数的亲 本配合力; 导入 RM258-165 bp 条带改良其千粒重的 亲本配合力。可以针对具体目标有选择地导入有利 等位变异进行改良, 但是在改良之前必须选择有代 表性且配合力高的不育系作参照。反之亦然。

本研究依据标记基因型“杂合组”和“纯合组”计 算的亲本性状配合力概念与传统的一般配合力概念 略有不同。这里是指两类亲本间的配合力, 而这两 类亲本是根据篮选到的亲本配合力标记基因型确定 的，我们称之为标记基因型配合力。在使用标记基 因型配合力时，应该注意 NCII 组合数据必须完整; 当纯合组性状平均值与杂合组性状平均值 $t$ 测验呈 现极显著差异 $(P<0.01)$ 时, 还必须注意纯合位点和 杂合位点的组合数量越接近越好。满足这些条件的 优异等位变异组合才是有效的。 


\section{4 结论}

共发现 20 个 SSR 标记基因型与亲本产量及其 构成性状优异配合力显著相关, 可直接将其用于粳 稻恢复系配合力的分子标记辅助改良。

\section{References}

[1] Deng H-F(邓华凤), He Q(何强). Status and technical strategy on development of japonica hybrid rice in china. Hybrid Rice (杂交 水稻), 2006, 21(1): 1-6 (in Chinese with English abstract)

[2] Yang Z-Y(杨振玉), Chen Q-B(陈秋柏), Chen R-F(陈荣芳). The breeding of japonica rice restorer C57. Acta Agron Sin (作物学 报), 1981, 7(8): 153-156 (in Chinese with English abstract)

[3] Yang Z-Y(杨振玉), Chen Q-B(陈秋柏), Chen R-F(陈荣芳). The breeding of japonica hybrid rice Liyou57. Sci Agric Sin (中国农 业科学), 1982,15 (1): 38-42 (in Chinese with English abstract)

[4] Lu Z-M(陆作楣). On the combining ability selection in hybrid rice breeding. Chin J Rice Sci (中国水稻科学), 1999, 13(1): 1-5 (in Chinese with English abstract)

[5] Lu Z-M(陆作楣), Tao J(陶瑾). Study of combining ability in hybrid rice of three lines lection and selection of combination. Seed (种子), 1986, (4): 24-28 (in Chinese)

[6] Hong D-L(洪德林), Pan E-F(潘恩飞), Chen C-Q(陈长青). Comparative studies on harvest index between hybrids and pure lines in japonica rice (Oryza sativa L.). J Nanjing Agric Univ (南 京农业大学学报), 1998, 21(4): 12-18 (in Chinese with English abstract)

[7] Hong D-L(洪德林), Yang K-Q(杨开晴), Pan E-F(潘恩飞). Heterosis of $F_{1}$ s derived from different ecological types and combining ability of their parents in japonica rice (Oryza sativa L.). Chin J Rice Sci (中国水稻科学), 2002, 16(3): 216-220 (in Chinese with English abstract)

[8] Leng Y(冷燕), Hong D-L(洪德林). Quality trait of hybrid rice grain derived from different ecological type and their genetic analysis in japonica rice (Oryza sativa L.). Chin J Rice Sci (中国 水稻科学), 2004, 18 (1): 29-33 (in Chinese with English abstract)

[9] Li J-H(李建红), Hong D-L(洪德林). Combining ability analysis of main agronomic and quality traits of BT type CMS lines newly bred in japonica rice. J Nanjing Agric Univ (南京农业大学学报), 2004, 27(4): 11-16 (in Chinese with English abstract)

[10] Li J-H(李建红), Hong D-L(洪德林). Combining ability for agronomic and quality traits of BT type iso-cytoplasmic restorer lines newly bred in japonica rice (Oryza sativa L.). Acta Agron Sin (作物学报), 2005, 31(7): 851-857 (in Chinese with English abstract)

[11] Jin W-D(金伟栋), Zhang W(张旺), Hong D-L(洪德林). Heterosis of late-maturing japonica hybrids (Oryza sativa L.) and parent's combining ability in south Jiangsu region. Acta Agron Sin (作物学报), 2005, 31(11): 1478-1484 (in Chinese with English abstract)

[12] Yuan L(袁玲), Zhu L-L(祝莉莉), He G-C(何光存). Localization of genes controlling rice quality traits with SSR markers. Wuhan Univ $J$ (Nat Sci Edn)(武汉大学学报·理学版), 2002, 48(4): 507-510 (in Chinese with English abstract)

[13] Ni D-H(倪大虎), Yi C-X(易成新), Yang J-B(杨剑波). Developing rice lines resistant to bacterial blight and blast with molecular marker-assisted selection. Acta Agron Sin (作物学报), 2008, 34(1): 100-105 (in Chinese with English abstract)

[14] Wang Y(王岩), Fu X-M(付新民), Gao G-J(高冠军), He Y-Q(何 予卿). Improving the e grain quality of Minghui 63, a restorer line of rice with good quality through marker-assisted selection. Mol Plant Breed (分子植物育种), 2009, 7(4): 661-665 (in Chinese with English abstract)

[15] Liu X C, Wu J L. SSR heterogenic patterns of parents for marking and predicting heterosis in rice breeding. Mol Breed, 1998, 4: 263-268

[16] Liu X C, Ishiki K, Wang W X. Identification of favorable AFLP markers to heterosis in hybrid rice. Breed Sci, 2002, 52: 201-206

[17] Liu X C, Chen S C, Chen J S, Ishiki K, Wang W X, Yu L Q. Improvement of combining ability for restorer lines with identified SSR marker in hybrid rice breeding. Breed Sci, 2004, 54: 341-346

[18] Liu X-C(刘小川), Wang W-X(王渭霞). Identification of molecular markers referred to the grain quality traits of rice hybrids based on the combining ability of their parental lines. Chin J Rice Sci (中国水稻科学), 2005, 19(1): 25-28 (in Chinese with English abstract)

[19] Cheng B-S(程宝山), Wan Z-B(万志兵), Hong D-L(洪德林). Establishment of SSR fingerprint map and analysis of genetic similarity among 35 varieties in japonica rice (Oryza sativa L.). $J$ Nanjing Agric Univ (南京农业大学学报), 2007, 30(3): 1-8 (in Chinese with English abstract)

[20] Shi K-Y(时宽玉), Hong D-L(洪德林). Polymorphism of 6 hybrid ids and their parents amplified by SSR primers and its application in rice (Oryza sativa L.). J Nanjing Agric Univ (南京农业大学学 报), 2005, 28(4): 1-5 (in Chinese with English abstract)

[21] Mu P(穆平), Zhang H-L(张洪亮), Jiang D-F(姜德峰), Liu L-F(刘立峰), Li Z-C(李自超). QTL mapping and interactions between QTL and environment for yield and its components using a DH population derived from a lowland and upland rice Cross. Sci Agric Sin (中国农业科学), 2005, 38(9): 1725-1733 (in Chinese with English abstract)

[22] Ye S-P(叶少平), Zhang Q-J(张启军), Li J-Q(李杰勤), Zhao $\mathrm{B}$ (赵兵), Li P(李平). QTL mapping for yield component traits using (Pei'ai 64s/Nipponbare) $\mathrm{F}_{2}$ population. Acta Agron Sin (作 物学报), 2005, 31(12): 1620-1627 (in Chinese with English abstract)

[23] Xu J-L(徐建龙), Xue Q-Z(薛庆中), Luo L-J(罗利军), Li Z-K(黎 志康). QTL dissection of panicle number per plant and spikelet number per panicle in rice (Oryza sativa L.). Acta Genet Sin (遗 传学报), 2001, 28(8): 752-759 (in Chinese with English abstract)

[24] Xing Y-Z(邢永忠)，Xu G-C(徐才国)，Hua J-P(华金平), Tan $\mathrm{Y}-\mathrm{F}($ 谈移芳). Analysis of QTL $\times$ Environment interaction for 
rice panicle characteristics. Acta Genet Sin (遗传学报), 2001, 25(5): 439-446 (in Chinese with English abstract)

[25] Xu L(许凌), Zhang Y-D(张亚东), Zhu Z(朱镇), Wang C-L(王才 林). Dissection of QTLs in two year s for yield component traits in rice (Oryza sativa L.). Chin J Rice Sci (中国水稻科学), 2008, 22(4): 370-376 (in Chinese with English abstract)

[26] Guo L-B(郭龙彪), Luo L-J(罗利军), Xing Y-Z(邢永忠), Xu G-C(徐才国). Dissection of QTLs in two years for important agronomic traits in rice (Oryza sativa L.). Chin J Rice Sci (中国水 稻科学), 2003, 17(3): 211-218 (in Chinese with English abstract)

[27] Ma D-P(马大鹏), Luo L-J(罗利军), Wang C-Y(汪朝阳), He Y-Q(何予卿). Mapping QTLs for yield and its component traits of rice using a recombinant inbred line population. Mol Plant Breed (分子植物育种), 2004, 2(4): 507-512 (in Chinese with English abstract)

[28] Li S-B(李绍波), Yang G-H(杨国华), Zhang Z-H(章志宏), Zhu Y-G(朱英国). Mapping of QTL controlling yield component related traits in rice. Wuhan Univ J (Nat Sci Edn)(武汉大学学 报·理学版), 2008, 54(6): 713-718 (in Chinese with English abstract)

[29] Du J-H(杜景红), Fan Y-Y(樊叶杨), Wu J-R(吴季荣), Zhuang J-Y(庄杰云). Dissection of QTLs for yield traits on the short arm of rice chromosome 6. Sci Agric Sin, 2008, 41(4): 939-945 (in Chinese with English abstract)

[30] Mu P(穆平), Huang C(黄超), Li J-X(李君霞), Liu L-F(刘立峰), Liu Y-J(刘式菊), Li Z-C(李自超). Yield trait variation and QTL mapping in a $\mathrm{DH}$ population of rice under phosphorus deficiency. Acta Agron Sin (作物学报), 2008, 34(7): 1137-1142 (in Chinese with English abstract)

[31] Zhao X Q, Xu J L, Zhao M, Lafitte R, Zhu L H, Fu B Y, Li Z K. QTLs affecting morph-physiological traits related to drought tolerance detected in overlapping introgression lines of rice (Oryza sativa L.). Plant Sci, 2008, 174: 618-625

[32] Brondani C, Rangel P, Brondani R, Ferreira M. QTL mapping and introgression of yield related traits from Oryza glumaepatula to cultivated rice (Oryza sativa L.) using micro satellite markers. Theor Appl Genet, 2002,104: 1192-1203

[33] Yoon D B, Kang K H, Kim H J, Ju H G, Kwon S J, Suh J P, Jeong Q Y, Ahn S N. Mapping quantitative trait loci for yield components and morphological traits in an advanced backcross population between Oryza grandiglumis and the O. sativa japonica cultivar Hwaseongbyeo. Theor Appl Genet, 2006, 112: 1052-1062

[34] Mo H-D(莫惠栋). The analysis of combining ability in $p \times q$ mating pattern. J Jiangsu Agric Coll (江苏农学院学报), 1982, 3(3): 51-57 (in Chinese)

[35] Mo H-D(莫惠栋). The analysis of combining ability in $p \times q$ mating pattern (continued). J Jiangsu Agric Coll (江苏农学院学报), 1982, 3(4): 53-57 (in Chinese)

[36] Xu C-W(徐辰武), Zhang A-H(张爱红), Zhu Q-S(朱庆森). Genetic analysis of quality characters of rice grain in indica-japonica hybrids. Acta Agron Sin (作物学报), 1996, 22(5): 530-534 (in Chinese with English abstract)

[37] Gu F-L(谷福林), Su Z-Q(苏自强), Wang S-F(王水方). 86 You 8, a new fine quality japonica hybrid rice combination. Hybrid Rice (杂交水稻), 2001, 16(2): 59-60 (in Chinese) 